\title{
Correction to: The Expression of Immunomodulation-Related Cytokines and Genes of Adipose- and Bone Marrow-Derived Human Mesenchymal Stromal Cells from Early to Late Passages
}

\author{
Chin Hee Mun ${ }^{1,2,3} \cdot$ Mi-Il Kang ${ }^{4}$ Yong Dae Shin ${ }^{1,2} \cdot$ Yeseul Kim $^{1} \cdot$ \\ Yong-Beom Park ${ }^{1,2,3}$
}

Published online: 12 January 2019

(c) The Korean Tissue Engineering and Regenerative Medicine Society and Springer Nature B.V. 2019

Correction to: Tissue Eng Regen Med (2018) 15(6):771-779

https://doi.org/10.1007/s13770-018-0147-5

Unfortunately, the online published article has error in Figure 3. The name of protein, "Gelectin-1" should be corrected to "Galectin-3". The corrected Fig. 3 is placed in the following page.

The original article can be found online at https://doi.org/10.1007/s13770-018-0147-5.

Yong-Beom Park

yongbpark@yuhs.ac

1 Division of Rheumatology, Department of Internal Medicine, and Department of Medical Sciences, Institute for

Immunology and Immunological Disease, Yonsei University

College of Medicine, 50 Yonsei-ro, Seodaemun-gu,

Seoul 03722, Republic of Korea

2 BK21 Plus Project, Department of Medical Sciences, Yonsei University College of Medicine, 50 Yonsei-ro, Seodaemungu, Seoul 03722, Republic of Korea

3 Severance Biomedical Science Institute, Yonsei University College of Medicine, 50 Yonsei-ro, Seodaemun-gu, Seoul 03722, Republic of Korea

4 Division of Rheumatology, Department of Internal Medicine, Dankook University College of Medicine, 201 Manghyangro, Dongnam-gu, Cheonan, Chung Nam, Republic of Korea 


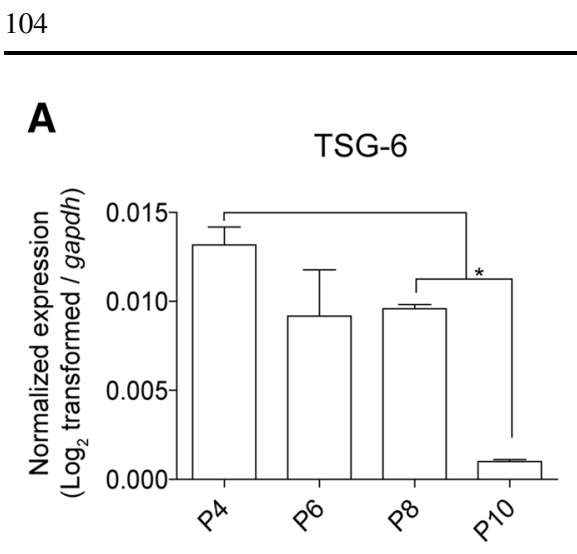

B

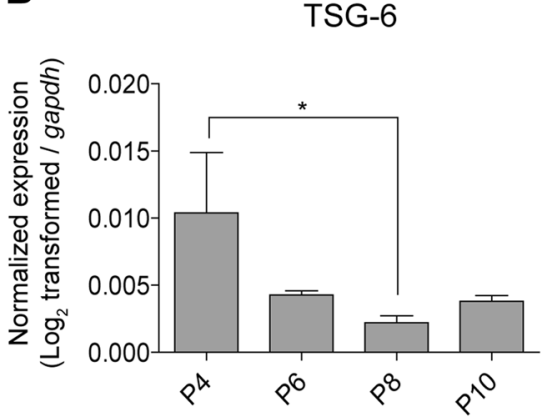

E

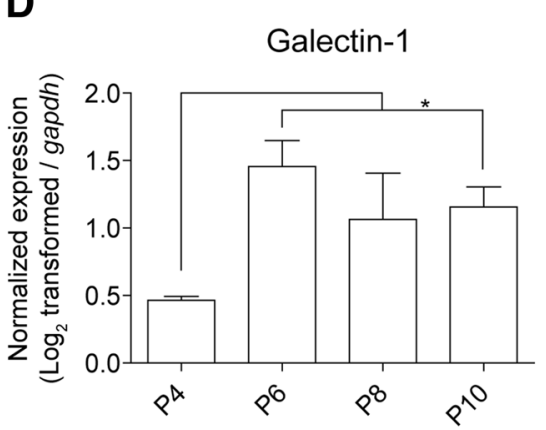

G

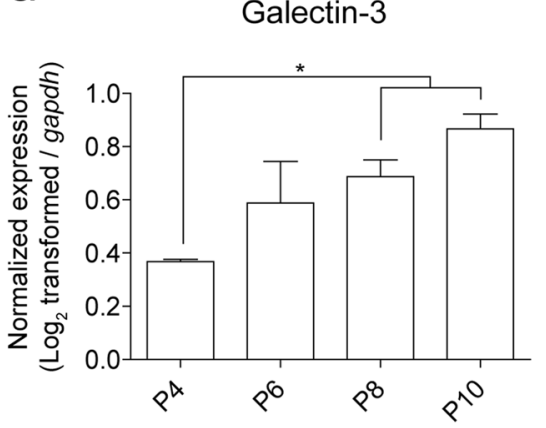

J
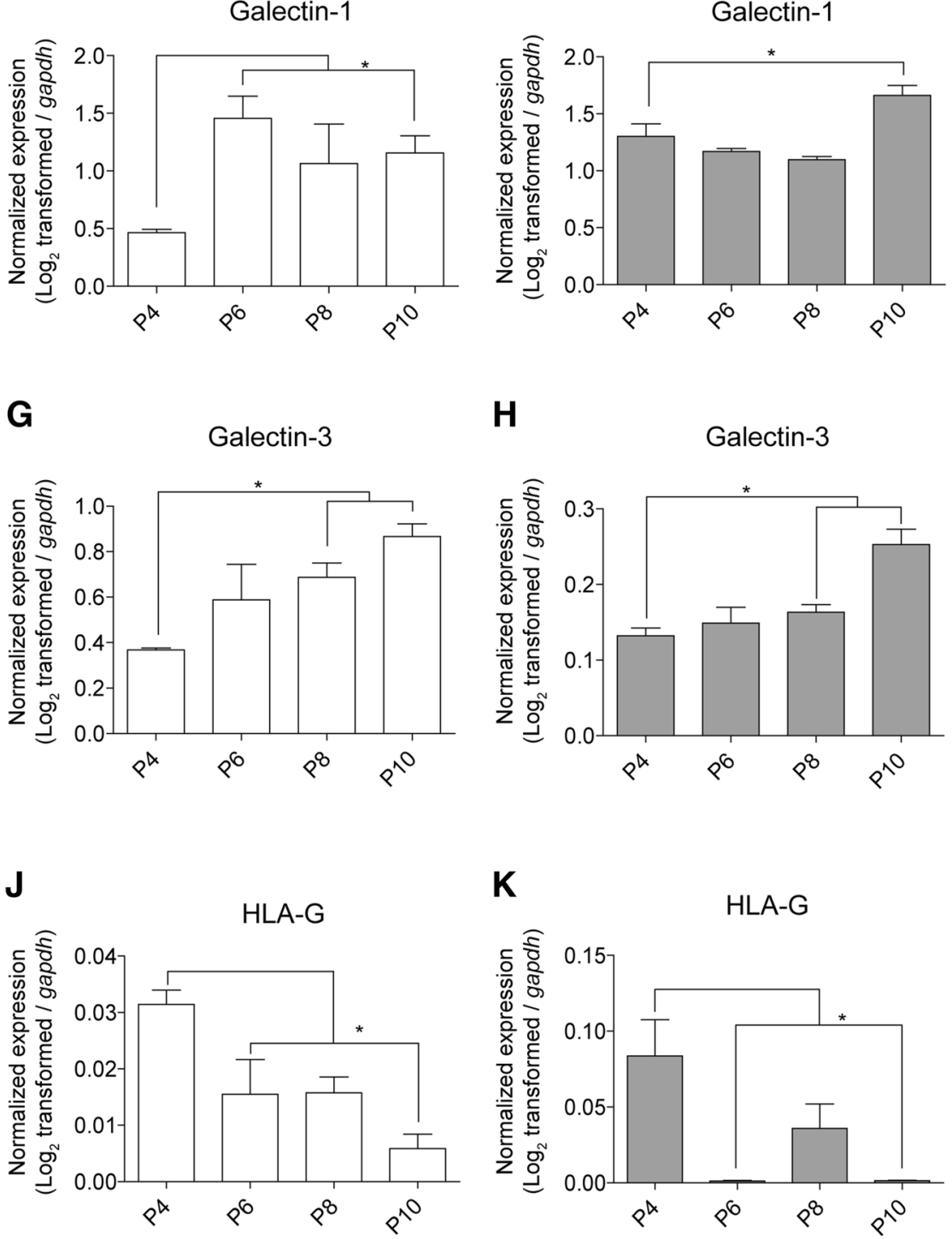

HLA-G

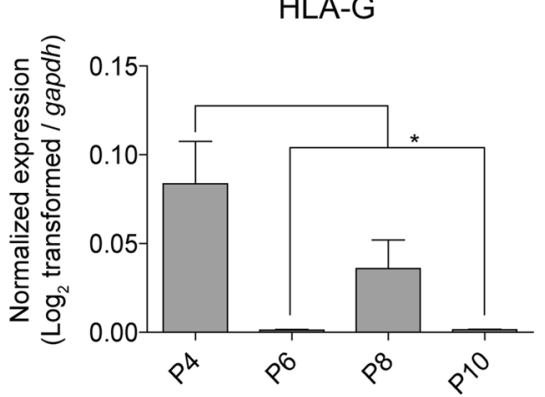

Tissue Eng Regen Med (2019) 16(1):103-105

C

TSG-6

- $A D$

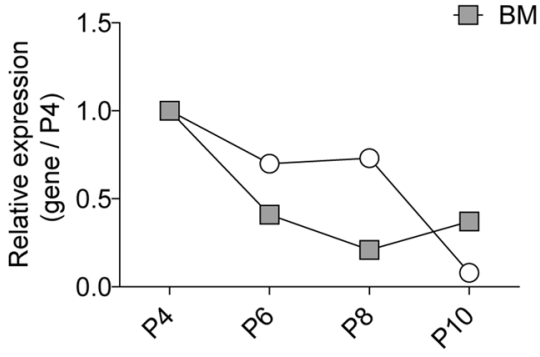

F

Galectin-1

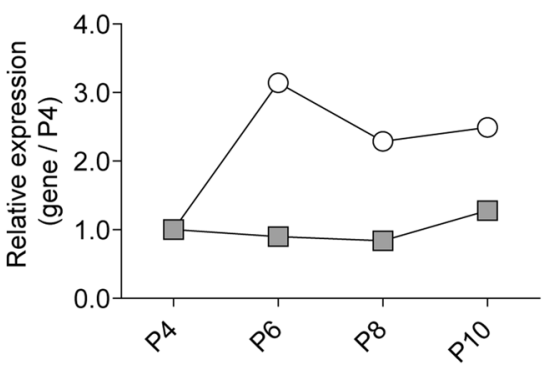

Galectin-3

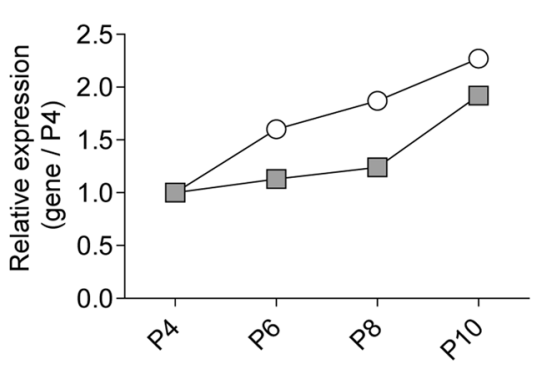

L

HLA-G

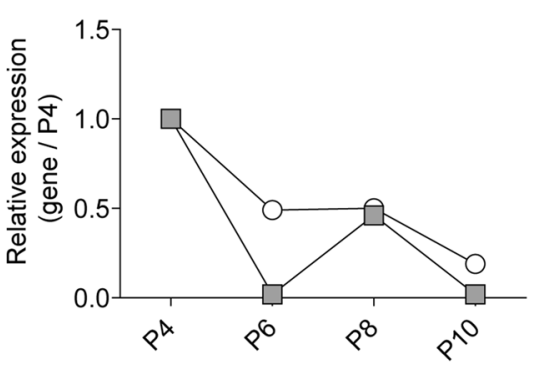

Springer 
4 Fig. 3 Gene expressions relevant to immune modulation of AD- and BMMSCs through passages. A-L TSG-6 and HLA-G expressions were decreased, whereas galectin- 1 and galectin- 3 expressions were increased or maintained throughout passages in AD-MSCs and BM-MSCs. Data are shown as normalized gene expression $(\mathbf{A}-\mathbf{B}, \mathbf{D}-\mathbf{E}, \mathbf{G}-\mathbf{H}, \mathbf{J}-\mathbf{K})$ compared to the housekeeping gene, GAPDH, and as relative gene expression $(\mathbf{C}, \mathbf{F}, \mathbf{I}$, L) compared to AD- and BM-MSC P4. Each experiment was conducted in triplicate. All values are the mean \pm SEM. $* p<0.05$ was considered statistically significant versus $\mathrm{P} 4$ of each origin, ns means not significant 